\title{
Effects of LC-PUFA (long chain poly unsaturated fatty acids) in infancy
}

\begin{abstract}
For human every nutrient is very important, but for infants, some nutrient plays an extraordinary role in their development. LC-PUFA is one of them. It is the combined form of ALA and LA essential fatty acids containing long chain carbons along with two or more double bonding and D6D is the regulator enzyme to combine them. The requirement of LC-PUFA is a little but it is essential for optimal development of infant which can be possible by adding in its formulas. Breast milk of human is a perfect source of it. Through research, it has been proved that requiring an amount of LCPUFA can be fulfilled by breast milk but the actual amount of it depends on mothers' diet habit. There are some other sources like meat, fish oil, egg, and seaweed. The LC-PUFA is mainly necessary for infant growth, Eye function, Play a role on cognitive function, behavior developments and also essential for cellular, membrane and brain development. If an infant cannot get the proper amount of LC-PUFA from breast milk, required amount can be added it to the formula of the infant.
\end{abstract}

Volume 7 Issue 4 - 2017

\author{
Sarwar Inam AKM, Suzauddula \\ Department of Nutrition and Food Engineering, Daffodil \\ International University, Bangladesh
}

\begin{abstract}
Correspondence: Sarwar Inam AKM,Assistant Professor, Department of Nutrition and Food Engineering, Daffodil International University, Bangladesh, Tel +8801717337817, Email inam.nfe@diu.edu.bd
\end{abstract}

Received: October 29, 2017 | Published: November 13, 2017

Keywords: LC-PUFA; infancy, infant, polyunsaturated fatty acid

Abbreviations: LCPUFA, long-chain polyunsaturated fatty acids; EPA, eicosapentaenoic acid; DHA, docosahexaenoic acid; AA, arachidonic acid; ALA, alfa linolenic acid; LA, linoleic acid; D6D, delta 6 desaturases; ADHD, attention deficit hyperactive disorder

\section{Introduction}

LC-PUFA (Long-chain polyunsaturated fatty acids) is those polyunsaturated fatty acids which have more than 20 carbon atoms and 2 or more double bindings. Most important LC-PUFA are EPA or Eicosapentaenoic acid (C 20:5 n-3), AA or Arachidonic acid (C 20:4 n-6) and DHA or Docosahexaenoic Acid (C 22:6 n-3). LC-PUFA is important for the structural and functional development of an infant. Such as lower intake of DHA has a positive correlation with the risk of proper neurological development. And the deficiency of DHA and EPA may cause visual problems and allergic diseases. Infants can perform endogenous conversion very low amount from 18 carbon precursor fatty acids such as linoleic acid and alfa-linolenic acid to LCPUFA. So they have to be depended on antenatal placental transfer, breast milk or infant formula for LC-PUFA. Several types of research showed that in the postnatal period LC-PUFAs supplementation with infant formula or with lactating mother's diet or the direct addition to the breast milk is adequate and practical to increase DHA level of infants. ${ }^{1}$ Supplementation of LC-PUFA as an infant formula can be very much helpful if there is any shortage of LC-PUFA in infants. It is always better to give the lactating mother foods containing LC-PUFA rather than to feed infants to infant formula.

\section{Discussion}

\section{LC-PUFA metabolism}

In the human body, essential fatty acids such as the alfa-linolenic acid (ALA, omega-3) and linoleic acid (LA, omega-6) convert to LC-PUFA through enzymatic desaturation and chain elongation (Figure 1). LC-PUFA expose a considerable perinatal aggregation in membrane-rich tissues e.g. brain and retina. ${ }^{2}$

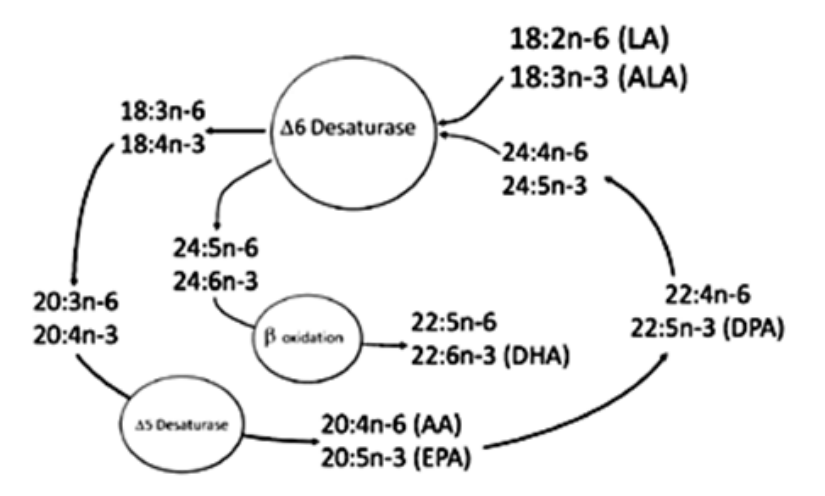

Figure I Metabolism of linoleic acid (I8:2n-6) and linolenic acid (I8:3n-3) to long-chain polyunsaturated fatty acids (LC-PUFA). ${ }^{2}$

For the omega- 6 series conversion of LA to the 20-carbon arachidonic acid (AA, 20:4 n-6) occurs and for the omega-3 series, ALA converts to 20-carbon fatty acid EPA (20:5 n-3) and a 22-carbon fatty acid DHA (22:6 n-3). It is remembered that only one set of enzyme works here and both fatty acids compete to use that enzyme. And delta 6 desaturases (D6D) is regarded as the main regulator enzyme as it works twice in the conversion of ALA to DHA. ${ }^{3}$ But this conversion occurs in very little amount for the infants. That is why it is recommended to feed them LC-PUFA directly so that there will be no deficiency in their body.

\section{LC-PUFA in infant formula}

The level of LC-PUFA is zero in infant means the alteration of essential fatty acid to LC-PUFA is not sufficient. This can be observable from the level of plasma and red blood cells. Normally it is not important to add LC-PUFA in infant formula but many research showed that the optimal development of infant can be possible by adding LC-PUFA in infant formulas. In accordance with EU directive 2006/141, LC-PUFA is allowable to add in infant formulas. ${ }^{4}$ Infant 
formula should contain at least $0.3 \%$ DHA and $0.35 \%$ AA of total fatty acid but for preterm infants, the infant formula should comprise of $0.35 \%$ of DHA and $0.4 \%$ of AA of total fatty acids. For lactating women should be recommended those food sources containing LCPUFA. ${ }^{5}$ A balanced diet containing necessary amount of AA and DHA will not show any poor growth or adverse effect if that diet is made with sufficient antioxidant protection. ${ }^{6}$

\section{Sources of LC-PUFA}

Human breast milk contains averagely $0.4 \%$ AA of total fatty acids and $0.2 \%$ DHA of total fatty acids though it depends on mother's diet. Fatty acid profile of human milk during the first year of lactation is showed in the Figure 2.

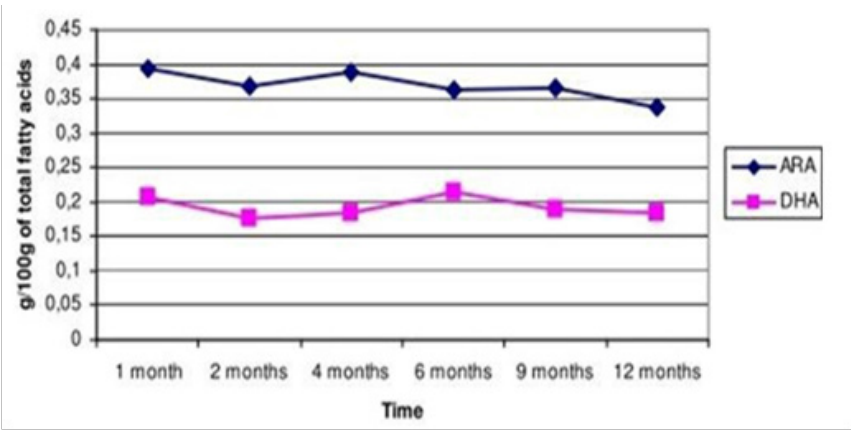

Figure $\mathbf{2}$ Level of Arachidonic acid and Docosahexaenoic acid in breast milk ( ${ }^{\text {st }}$ year of lactation).

Beside human milk LC-PUFA can be found in the following foods:
a. Meat (Main source for AA): AA in pork liver $(870 \mathrm{mg} / 100 \mathrm{~g}$ )
b. Fish oil (Main source for DHA): AA in bonito $(280 \mathrm{mg} / 100 \mathrm{~g}$ )
c. Eggs: AA in egg yolk $(297 \mathrm{mg} / 100 \mathrm{~g})$
d. Certain vegetable oils: AA in linseed oil $(500 \mathrm{mg} / 100 \mathrm{~g})$
e. Seaweed: AA in red algae.

\section{Benefits of LC-PUFA}

Though human milk contains LC-PUFA, it can vary due to mother's diet and lifestyle. DHA is available in the brain and retina. DHA and AA are the precursors of eicosanoid which is a very important component of membrane lipids. Eicosanoids control the cellular and organelle integrity and necessary intracellular mediator for gene expression. ${ }^{7}$ LC-PUFAs are very important to the infant for visual, brain and cognitive development. Other benefits may be included as motor development, lower blood pressure and modulate the immune response. Main three benefits are discussed below.

Effect on infant growth: Report showed that the birth weight of the infant is related to mother's consumption of fish. Here fish is one of the main sources of DHA. It also has been reported that infant growth and blood levels of AA are correlated. In Figure 3 the correlation between levels of AA and DHA in the breast milk and weight gain of 3 months infant in China has been showed. Not only weight but also the length of the infants is correlated with the DHA of their mothers' milk (Figure 4). ${ }^{8}$

Effects on visual function: Makrides et al., ${ }^{9}$ showed in a report that an infant formula with $0.36 \%$ DHA, $0.58 \%$ EPA and $0.27 \% \gamma$-linolenic acid exhibits better VEP (Visual evoked potential) acuity than control infant formula without DHA. That report also demonstrated that no visual acuity occurred between those infants fed with DHA enriched infant formula and those fed only breast milk. Birch et al., ${ }^{10}$ also made an experiment with two types of infant formula: one with $0.35 \%$ DHA another with $0.72 \% \mathrm{AA}$ and $0.36 \%$ DHA. Both formulas gave better VEP acuity at 6,17 and 52 weeks ages.
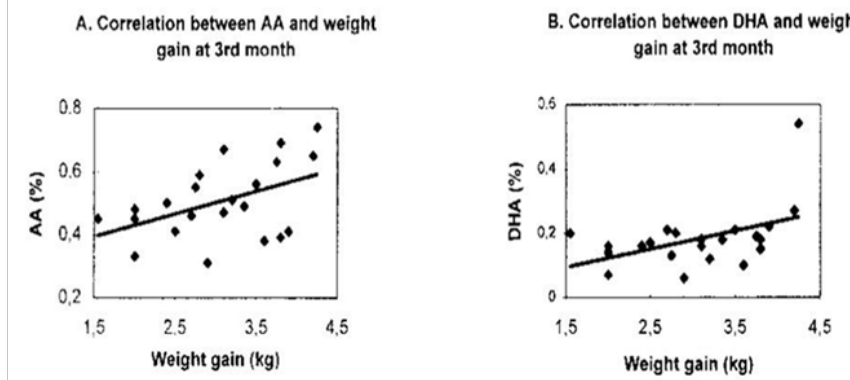

Figure 3 Correlation between levels of AA and DHA in breast milk and weight gain of 3 months of age in Chinese infants. ${ }^{8}$
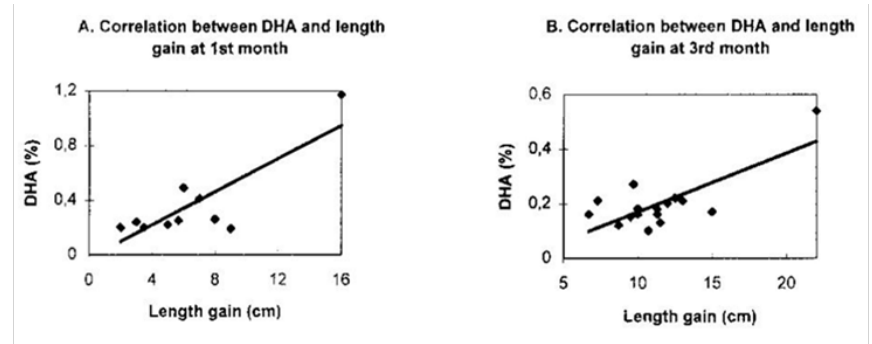

Figure 4 Correlation between levels of DHA in breast milk and length gain of Imonth and 3months Chinese infants. ${ }^{8}$

Effects on cognitive function: Most of the research based on the cognitive function which is developed by DHA and AA mainly concentrated on pre-term infants. DHA and AA showed the helpful effect on mental development and problem-solving ability. EPA and DHA from fish and fish oil show beneficial effects on neuropsychiatric disorders. Decrease amount of LC-PUFAs in blood cells causes different types of neuropsychiatric conditions like Alzheimer's disease, schizophrenia and depression in elderly ages. So infant formula enriched with LC-PUFA is very much necessary. ${ }^{11}$

Effects on behaviour: Several types of research have been done in this area but most of them are highly speculative. Some behavioral disorders such as attention deficit hyperactive disorder (ADHD), autism, dyspraxia and dyslexia are related to the problem in fatty acid metabolism. Inefficient conversion of the essential fatty acid to long chain EPA and DHA is responsible for these. It has been reported that infants with ADHD diseases showed improve result after giving them $500 \mathrm{mg}$ per day LC-PUFA containing DHA and EPA as a supplement. ${ }^{11}$

\section{Recommendation for addition of LC-PUFA in infant formula}

I. LC-PUFA can be added to the infant formulas under the following recommendation.

II. The added DHA should be above $0.2 \%$ and should be below $0.5 \%$ of the fatty acids. The maximum level of other omega- 3 LC-PUFAs should be limited to $1 \%$.

III. EPA will be always lower than DHA.

IV. The omega-6 LC-PUFA should be limited to $2 \%$ of which AA will be maximum $1 \%$. AA should be equal or higher than DHA. 


\section{Conclusion}

Most of the studies have proved that there is some strong correlation between LC-PUFA (mainly EPA, DHA and AA) and infants' growth, visual, cognitive and behavioral functions. Recommended doses are the very important matter here, especially for brain development. The correct balance between the precursors LA and ALA and LC-PUFA (AA and DHA) is also necessary. It has been reported that breastfed infants have a higher amount of DHA in their brain cortex compared to those fed infant formulae without DHA. ${ }^{12,13}$ The relationship between dose and effect is the very important term here.

Preformed LC-PUFA can be found in breast milk. And the level of LC-PUFA in plasma and tissue phospholipids is always high for the breast-fed infant compared to infant formula-fed infants. But to be in safe side it is always recommended to supplement the infant formulas enriched with LC-PUFA. Not only for infant formulas but also during pregnancy and lactation period mother should be provided sufficient amount of LC-PUFA enriched foods to avoid any occurrence of LCPUFA depletion.

\section{Acknowledgements}

The authors thank the Dept. of Nutrition and Food Engineering and the Library of Daffodil International University for all kind of academic supports. Authors also acknowledge Dr. Md. Bellal Hossain, Head, Dept. of Nutrition of Food Engineering, Daffodil International University for his help and support.

\section{Conflict of interest}

The author declares no conflict of interest.

\section{References}

1. Meldrum SJ, D’Vaz N, Casadio Y, et al. Determinants of DHA levels in early infancy: differential effects of breast milk and direct fish oil supplementation. Prostaglandins Leukot Essent Fatty Acids. 2012;86(6):233-239.
2. Koletzko B, Rodriguez-Palmero M. Physiological aspects of human milk lipids and implications for infant feeding: a workshop report. Acta Paediatr. 2001;100(11):1405-1415.

3. Gibson RA, Muhlhausler B, Makrides M. Conversion of linoleic acid and alpha-linolenic acid to long-chain polyunsaturated fatty acids (LCPUFAs), with a focus on pregnancy, lactation and the first 2years of life. Matern Child Nutr. 2011;7(Suppl 2):17-26.

4. European Union. Commission directive 2006/141/EC of 22 December 2006 on infant formula and amending Directive 1999/21/EC. Official Journal of the European Union. 2006.

5. Koletzko B, Agostoni C. Long chain polyunsaturated fatty acids (LCPUFA) and perinatal development. Acta Paediatrica. 2001;90(4):460464.

6. Agostoni C, Trojan S, Bellù R, et al. Neurodevelopmental quotient of healthy term infants at 4 months and feeding practice: The role of longchain polyunsaturated fatty acids. Pediatr Res. 1995;38(2):262-266.

7. Santos FS, Chaves CR, Costa RS, et al. Status of cis and trans fatty acids in Brazilian adolescent mothers and their newborns. J Pediatr Adolesc Gynecol. 2012;25(4):270-276.

8. Xiang M, Zetterström R. Relation between polyunsaturated fatty acids and growth. Acta Paediatr Suppl. 1999;88(430):78-82.

9. Makrides M, Neumann M, Simmer K. Are long-chain polyunsaturated fatty acids essential nutrients in infancy? Lancet. 1995;345(8963):14631468.

10. Birch EE, Hoffman DR, Uauy R, et al. Visual acuity and the essentiality of docosahexaenoic acid and arachidonic acid in the diet of term infants. Pediatr Res. 1998;44(2):201-209.

11. Lunn J, Theobald HE. The health effects of dietary unsaturated fatty acids. Nutrition Bulletin. 2006;31(3):178-224.

12. Makrides M, Neumann MA, Byard RW. Fatty acid composition of brain, retina, and erythrocytes in breast- and formula-fed infants. Am J Clin Nutr. 1994;60(2):189-194.

13. Mitoulas LR, Kent JC, Cox DB. Variation in fat, lactose and protein in human milk over $24 \mathrm{~h}$ and throughout the first year of lactation. Br J Nutr. 2002;88(1):29-37. 